\title{
Effect of Deep Brain Stimulation on Vocal Motor Control Mechanisms in Parkinson's
}

\section{Disease}

\author{
Roozbeh Behroozmand ${ }^{1 *}$, Ph.D., Karim Johari ${ }^{1}$, M.Sc., Ryan M. Kelley ${ }^{2,3}$, B.Sc., Efthymia C. \\ Kapnoula $^{4}$, Ph.D., Nandakumar S. Narayanan ${ }^{5}$, M.D., Ph.D., Jeremy D.W. Greenlee ${ }^{6}$, M.D. \\ ${ }^{1}$ Speech Neuroscience Lab, Department of Communication Sciences and Disorders, University \\ of South Carolina, 915 Greene Street, Columbia SC 29028, USA \\ ${ }^{2}$ Medical Scientist Training Program, ${ }^{3}$ Program in Neuroscience University of Iowa, Iowa City, \\ IA 52242, USA \\ ${ }^{4}$ Basque Center on Cognition, Brain and Language, San Sebastian, Spain \\ ${ }^{5}$ Department of Neurology, University of Iowa, Iowa City, IA 52242, USA \\ ${ }^{6}$ Human Brain Research Lab, Department of Neurosurgery, University of Iowa, Iowa City, IA \\ 52242, USA
}

Running title: DBS effect on Parkinson's vocal motor control

*Corresponding author:

Roozbeh Behroozmand, Ph.D.

Assistant Professor

Department of Communication Sciences and Disorders

University of South Carolina

Email: r-behroozmand@sc.edu

Phone: (803)777-5055

Fax: (803)777-3081 


\begin{abstract}
Introduction: Deep brain stimulation (DBS) of the subthalamic nucleus (STN) is an effective treatment for limb motor symptoms in Parkinson's disease (PD); however, its effect on vocal motor function has yielded conflicted and highly variable results. The present study investigated the effects of STN-DBS on the mechanisms of vocal production and motor control.
\end{abstract}

Methods: A total of 10 PD subjects with bilateral STN-DBS implantation were tested with DBS ON and OFF while they performed steady vowel vocalizations and received randomized upward or downward pitch-shift stimuli ( \pm 100 cents $)$ in their voice auditory feedback.

Results: Data showed that the magnitude of vocal compensation responses to pitch-shift stimuli was significantly attenuated during DBS ON vs. OFF $(p=0.012)$. This effect was directionspecific and was only observed when subjects raised their voice fundamental frequency (F0) in the opposite direction to downward stimuli $(p=0.019)$. In addition, we found that voice F0 perturbation (i.e. jitter) was significantly reduced during DBS ON vs. OFF $(p=0.022)$, and this DBS-induced modulation was positively correlated with the attenuation of vocal compensation responses to downward pitch-shift stimuli $(r=+0.57, p=0.028)$.

Conclusions: These findings provide the first data supporting the role of STN in vocal F0 motor control in responses to altered auditory feedback. The DBS-induced attenuation of vocal compensation responses may result from increased inhibitory effects of the subcortical hyperdirect (fronto-subthalamic) pathways on the vocal motor cortex, which can help stabilize voice F0 and ameliorate vocal motor symptoms by impeding PD subjects' abnormal (i.e. overshooting) vocal responses to alterations in the auditory feedback.

Keywords: Parkinson's disease; Deep brain stimulation; Subthalamic nucleus; Voice motor control; Auditory feedback 


\section{Introduction}

Deep brain stimulation (DBS) of the subthalamic nucleus (STN) has long been established as a highly effective medical treatment for the limb motor symptoms in Parkinson's disease (PD) $[1,2]$. However, reports on the effect of STN-DBS on voice and speech have yielded considerably variable and sometimes contradicting results across individual subjects [3,4]. While a series of studies have documented that speech function was deteriorated in some subjects by the onset of dysarthria under STN-DBS [5-7], other investigators have reported amelioration of some oral motor and voice features, particularly improvements on vocal loudness and reduced glottal tremor following stimulation [8-11]. Despite these improvements on voice features, in most cases STN-DBS was reported to have an adverse effect on overall speech intelligibility [12], primarily because of the general dysarthrogenic impact of DBS on articulatory function in PD subjects. In addition, a recent study has suggested that STN-DBS significantly reduces the initial vowel formant space and it differentially affects vocal tract positions for sustained production of different vowel categories, corroborating the notion that articulatory gestures are constrained during speech under DBS [13]. However, speech deterioration was reported sporadically and varied significantly across individual subjects, suggesting that the observed dysarthrogenic effect under STN-DBS may be multi-factorial. For example, spread of stimulation current to adjacent neural pathways involved in speech motor control has been implicated, and studies have suggested that pre-surgical speech performance, active electrode location, and PD duration inform speech intelligibility outcomes after STN-DBS implantation [14-16]. Based on these factors, it is reasonable to assume that the conflicting results of STNDBS effect on voice vs. speech reflect the complexity of subcortical neural structures and their 
differential influence on motor control of phonatory (i.e. laryngeal) vs. articulatory mechanisms via subcortical-cortical interactions.

Recently, evidence from a number of studies has supported the notion that voice and speech impairment in PD is associated with deficits in neural mechanisms that are beyond the dedicated networks for phonatory or articulatory motor production [17-19]. In these studies, pitch and formant alterations were delivered real-time in the auditory feedback to probe the integrity of sensorimotor integration mechanisms for voice and speech production in subjects with PD. Findings of these studies revealed that PD is associated with deficits in sensorimotor integration mechanisms, and resulted in dysfunctions for incorporating auditory feedback to detect and correct for alterations (errors) in self-produced voice and speech. The sensorimotor deficits in PD were primarily characterized by subjects' abnormal (i.e. overshooting) compensatory vocal motor responses to pitch-shift alterations in the auditory feedback $[18,19]$, as well as their diminished functional capacity in generating adaptive motor responses to formant alterations in self-produced speech [17].

Findings of the previous studies have suggested that cortical-basal ganlia pathology can disrupt normal function and induce deficits in sensorimotor mechanisms of voice and speech in PD. One recent study [19] aimed to delineate the neuroanatomical bases of vocal sensorimotor impairment by recording neurophysiological responses to auditory feedback pitch alterations in PD during sustained vocalizations and has revealed pathological modulation of neural activities within a left-lateralized cortical network that involved areas in the superior and inferior frontal gyrus, premotor cortex, inferior parietal lobule, and the superior temporal gyrus. Although these findings provided supporting evidence for neurological impairments in the underlying sensorimotor networks of vocalization motor control, our understanding about the detrimental 
effects of PD on the mechanisms of voice and speech has remained largely elusive at the neural level, and therefore, further investigations are warranted for using advanced methodologies to elaborately examine the underlying mechanisms of voice and speech and their impairments in PD.

In the present study, we addressed the question as to how STN-DBS would modulate the underlying mechanisms of vocal production and motor control. We tested PD subjects with bilateral STN-DBS implantation under stimulation ON vs. OFF conditions while they produced steady vocalizations of a vowel sound and received altered auditory feedback (AAF) stimuli using randomized upward and downward pitch shifts at \pm 100 cents. Measures of vocal compensation responses to AAF were examined to determine the effect of STN-DBS on vocalization motor control. Based on findings of previous studies $[3,8]$, we hypothesized that STN-DBS would normalize deficits in vocal motor control mechanisms by counteracting and attenuating PD-related abnormal (i.e. overshooting) patterns of vocal compensation responses to pitch-shift alterations in the auditory feedback. Understanding the effect of STN-DBS on vocal motor control mechanisms will have important clinical implications for targeted treatment of voice motor symptoms in PD.

\section{Methods}

\subsection{Participants}

A total of 10 right-handed subjects diagnosed with idiopathic PD (4 females, mean age: 64.8 years, mean PD duration: 11.1 years) who received bilateral STN-DBS implantation participated in the present study. Subjects did not have any history of other neurological or psychiatric disorders, and completed extensive pre-surgical assessments including detailed neurological 
examinations, structural MRI, and neuropsychological evaluations that confirmed normal speech, language, and hearing functions. Subjects' demographic and clinical assessment data are summarized in Table 1. All subjects (except one) were tested ON-medication with their individually tailored dosages of dopaminergic medication to maximally reduce motor symptoms, and Levodopa Equivalent Dose (LED) was calculated for each subject. One subject (subject 2) was not taking any dopamine agonist medication due to side effects and his PD symptoms were well controlled with DBS alone with moderate settings at time of testing for this study (see Table 1). MDS-UPDRS Part III motor scores were assessed at time of testing with subjects ON their medications for STN-DBS ON and OFF conditions. When DBS was ON, all subjects were tested on their usual stimulation settings, as determined by their programming movement disorder specialist neurologist. The specialist also managed PD medication optimization through multiple clinic visits and DBS programming sessions to provide for the best overall motor function and minimization of treatment-related side effects in keeping with the best clinical practice standards. In addition, a survey of voice handicap index (VHI) was administered to assess subjects' perception of psychosocial consequences of their voice performance. All study procedures, including recruitment, data acquisition and informed consent were approved by the University of Iowa Institutional Review Board, and subjects were monetarily compensated for their participation.

\subsection{Experimental procedure}

The experiment was conducted in a sound attenuated booth in which subjects performed the experimental tasks in two blocks (DBS ON vs. OFF) that were counterbalanced across subjects. The time duration between DBS ON and OFF blocks was approximately 30 minutes, which let to 
approximately 1 hour for each subject to complete the experimental session for both blocks. During each block, subjects were instructed to repeatedly maintain steady vocalizations of the speech vowel sound /a/ at their conversational pitch and loudness for approximately 2-3 seconds while taking breaks between successive vocalizations. During each vocalization trial, a pitchshift stimulus altered the auditory feedback for $200 \mathrm{~ms}$ in the middle of vocalization with randomized onset delays at 750-1250 ms after the vocalization onset. The direction of stimuli was randomized between upward (+100 cents) and downward ( -100 cents) pitch shifts across vocalization trials within each block. A total of 150 vocalizations (75 per pitch-shift direction) were recorded during each block. Subjects' voice signal was picked up using head-mounted AKG condenser microphone (model C520), amplified by a Motu Ultralite-MK3 module, and was recorded at $44.1 \mathrm{KHz}$ during DBS ON and DBS OFF blocks. The auditory feedback was delivered through Etymotic insert earphones (model ER1-14A), and the timing, magnitude, direction and order of AAF stimuli were controlled by a custom-made program in Max 5.0 (Cycling '74) coupled with an Eventide Eclipse Harmonizer.

\subsection{Analysis of vocal responses}

Vocal acoustics including the fundamental frequency (F0), intensity, Harmonic to Noise Ratio (HNR), jitter (i.e. cycle-to-cycle voice F0 perturbation), and shimmer (i.e. cycle-to-cycle voice intensity perturbation) were extracted in Praat. In this analysis, jitter and shimmer were calculated as the average absolute difference between voice F0 (Hz) and/or voice intensity (dB) of consecutive cycles, respectively, according to the following formula:

$$
\text { jitter } / \text { shimmer }=\sum_{i=2}^{N}\left|T_{i}-T_{i-1}\right| /(N-1)
$$


where $T_{i}$ is the duration of the $i^{\text {th }}$ cycle and $N$ is the total number of cycles [20]. Using consistent methodologies implemented in previous studies of vocalization motor control during AAF [21], vocal compensation responses to pitch-shift stimuli were calculated in MATLAB by segmenting voice F0 contours into epochs from -100 to $800 \mathrm{~ms}$ relative to the onset of pitch-shift stimuli and then converting them from Hertz to Cents using the following formula:

$$
\text { Vocal Compensation }[\text { Cents }]=1200 \times \log _{2}\left(F 0 / F 0_{\text {Baseline }}\right)
$$

Here, $F O_{\text {Baselne }}$ is the mean pre-stimulus voice $\mathrm{F} 0$ at -100 to $0 \mathrm{~ms}$ before pitch-shift onset. Voice F0 contours in Cents were then averaged across all trials in each individual subject for upward and downward stimuli during DBS ON and DBS OFF conditions, separately. The grand-average profile of vocal compensation responses were calculated by averaging responses across all subjects for each stimulus direction and DBS condition, separately.

\subsection{Statistical analysis}

We conducted two-way repeated measures ANCOVAs to examine the effects of DBS (ON vs. OFF) and pitch-shift stimulus direction (up vs. down) on the magnitude of vocal compensation responses within a $200 \mathrm{~ms}$ time window centered on the peak. This time window was identified based on the profile of vocal compensation responses to AAF stimuli to capture the temporal dynamics of these responses and the effect of STN-DBS on modulating vocal motor behavior in response to upward and downward pitch-shift stimuli. The medication dose was controlled for by entering the subjects' LED as a co-variate in the statistical model and the effect size was estimated using partial Eta squared (partial $\eta^{2}$ ) in the ANCOVA model. Effects of DBS on voice F0, intensity, HNR, jitter, shimmer, VHI, and MDS-UPDRS Part III scores was examined using one-way ANCOVAs. Partial correlations with the LED effect being partialed out were 
performed to examine relationships between the modulation of vocal compensation magnitude and voice acoustics, as well as the VHI, UPDRS-III speech intelligibility scores, and clinical MDS-UPDRS measures of limb movement during DBS ON vs. OFF. In all statistical tests, the false discovery rate (FDR) method [22] was used to correct for multiple comparisons.

\section{Results}

\subsection{DBS effects on voice acoustics}

The effects of STN-DBS on acoustic measures of voice were examined in all subjects. In Fig. 1 (Panels A-C), the overlaid plots of voice F0, intensity, and HNR during DBS ON and OFF are shown for a representative subject. These plots suggest that the overall pattern of vocalization F0 was less variable (i.e. more stable) during DBS ON vs. OFF (Panel A, black versus red lines for the means and the corresponding shaded areas representing the standard errors). However, no such DBS-induced modulation effect was observed for voice intensity and HNR (Panels B and C). Panels A and B show that in this subject, voice F0 and intensity level were increased slightly throughout vocalization during DBS ON vs. OFF, but the measure of HNR remained relatively unchanged (Panel C). For the group data in all 10 subjects, results of the statistical analysis revealed a significant effect of DBS on voice jitter $\left(\mathrm{F}(1,8)=6.16, p=0.24\right.$, partial $\left.\eta^{2}=0.39\right)$, indicating reduced F0 perturbation during DBS ON vs. OFF; however, no such effect was observed for voice F0, intensity, HNR, and shimmer (Fig. 1, Panels D-H).

\subsection{DBS effects on vocal compensation}

In Fig. 2, results of the group analysis are shown for vocal compensation responses to upward (Panels A-C) and downward (Panels D-F) pitch-shift stimuli. The profiles of grand-average responses in panels $\mathrm{A}$ and $\mathrm{D}$ show that all subjects compensated for pitch shifts by changing their 
voice F0 in the opposite direction of the stimuli during both DBS ON and OFF conditions. However, an STN-DBS effect was evident as modulating vocal responses only to downward pitch-shift stimuli (Panel D). Indeed, statistical analyses revealed significant main effects of DBS $\left(\mathrm{F}(1,8)=10.38, p=0.012\right.$, partial $\left.\eta^{2}=0.57\right)$ and pitch-shift direction $(\mathrm{F}(1,8)=8.11, p=0.022$, partial $\left.\eta^{2}=0.51\right)$, as well as a significant DBS $\times$ pitch-shift direction interaction $(\mathrm{F}(1,8)=6.67, p=0.031$, partial $\eta^{2}=0.46$ ) on the magnitude of vocal compensation responses. Post-hoc analysis further confirmed the observed effects by showing that the magnitude of vocal compensation responses to downward pitch shifts was significantly decreased during DBS ON vs. OFF $(F(1,8)=8.49$, $p=0.019$, partial $\eta^{2}=0.52$ ), but no such DBS-induced modulation was observed for vocal compensation responses to upward stimuli (Fig. 2, Panels B and E). Since voice jitter was the only acoustic measure that showed a significant modulation by STN-DBS, we tested for correlation between jitter and vocal compensation magnitude and found a positive correlation $(\mathrm{r}=+0.57, p=0.028)$ only in response to downward pitch-shift stimuli (Fig. 2, Panels C and F). Notably, while all subjects showed a significant improvement in limb motor performance during DBS ON vs. OFF as indexed by the MDS-UPDRS Part III scores $(\mathrm{F}(1,8)=7.53, p=0.024$, partial $\eta^{2}=0.49$ ), this motor improvement was not correlated with modulation of vocal compensation responses to pitch-shift stimuli. In addition, no significant correlation was found between UPDRS-III speech intelligibility scores or subjective measures of VHI and modulation of vocal compensation responses to pitch alterations in the auditory feedback.

\section{Discussion}

The present study provided the first data examining the effects of STN-DBS on sensorimotor integration mechanisms of voice motor control. PD subjects with bilateral STN-DBS 
implantation were tested under an AAF paradigm to measure changes in their vocal compensation responses to pitch-shift alterations in the auditory feedback, as well as modulation of their voice acoustics during DBS ON vs. OFF conditions. For subjects' convenience during the experimental session, they were all tested while taking their normal PD medications on their scheduled times of the day (MED ON) to more closely replicate their 'real-world' condition while examining the effect of DBS on their vocal motor behavior. This condition was chosen based on evidence suggesting that PD medications do not significantly affect vocal motor function [23], and the DBS effect was further validated by controlling for the effect of medication dose as a co-variate in the statistical model during data analysis. Our data showed that STN-DBS resulted in a significant attenuation of the magnitude of vocal compensation responses to AAF in a direction-specific manner, which was only observed for compensatory responses that raised voice F0 in the opposite direction of downward pitch-shift stimuli. In addition, we found that STN-DBS was associated with a significant reduction in voice F0 perturbation (i.e. jitter), and this DBS-induced modulation was positively correlated with the attenuation of vocal compensation responses to downward pitch-shift alterations in the auditory feedback. These findings provide supporting evidence for the involvement of STN in regulating vocal production and motor control mechanisms and validate the effect of DBS on modulating these functions in subjects with PD.

PD subjects have been shown to exhibit deficits in vocal sensorimotor integration as indexed by their abnormally increased magnitude of compensatory responses to pitch-shift alterations in auditory feedback $[18,19]$. According to recent models of speech production [2426], motor control of vocalization is supported by sensorimotor integration mechanisms in the dorsal stream network that issue corrective feedforward motor commands in response to 
mismatch (error) between predicted (e.g., efference copies) and actual sensory feedback. In the context of these models, we suggest that the abnormal (i.e. overshooting) pattern of vocal compensation responses in PD can be explained by deficits in sensorimotor integration mechanisms of the dorsal stream network in one of the following ways: First, since both the auditory and somatosensory systems contribute to vocalization motor control, abnormal vocal compensation responses to pitch-shift errors in the auditory feedback in PD can be driven by cross-sensory dysfunction that leads to elevated sensory gain in the auditory system to compensate for reduced somatosensory sensation. This notion is supported by data showing reduced somatosensory sensitivity of laryngeal mucosa in PD [27], as well as increased vocal compensation responses to pitch-shift stimuli in healthy individuals with anesthetized vocal fold mucosa [28]. In the context of this notion, alterations in voice auditory feedback generate larger error signals that are transmitted from the sensory to motor regions, which subsequently lead to larger corrective motor commands for vocal compensation. Second, abnormal vocal compensation in PD may arise from sensorimotor integration deficits resulting from pathologically altered cortico-basal ganglia interactions. According to this notion, lack of dopaminergic input to neurons in the basal ganglia in PD can cause dysfunction in hyperdirect (fronto-subthalamic) and indirect (fronto-striatal-pallidal) pathways that play a crucial role in inhibiting motor responses during voluntary movement [29-31]. As a result, the reduced inhibitory output from the basal ganglia to cortical neurons within the dedicated networks of vocal motor control (e.g., inferior frontal gyrus, ventral premotor cortex, motor cortex) may drive abnormal compensatory efforts by generating overshooting motor responses to auditory feedback alterations in PD (Fig. 3). 
In addition, reduced inhibition from basal ganglia to cortical motor networks in PD may cause pathological increases in auditory neural sensitivity (or gain) to feedback errors that are controlled by top-down efference copy mechanisms during vocal production. This idea is supported by previous studies showing that auditory neural responses to pitch-shift alterations in voice feedback are enhanced during vocal production compared with passive listening in neurologically intact individuals $[32,33]$. This latter effect, also known as "Speech Perturbation Response Enhancement" or SPRE, has been incorporated into the recent State Feedback Control (SFC) model of speech [34], which posits that auditory feedback errors are used to correct the current estimates of vocal/articulatory states using a gain that determines how strongly feedback errors drive this state correction process. According to the SFC model, access to internal predictions through efference copies can increase the controlling gain during vocal production, and therefore, SPRE is associated with enhanced state correction responses to perturbations in the auditory feedback during vocalization vs. listening condition. In this context, disinhibition of top-down cortical motor mechanisms in PD may result in higher gains in the state correction process, which subsequently contribute to the generation of abnormally larger compensation responses to feedback error during vocal production.

Recent evidence has suggested that abnormal compensation behavior in PD is not driven by cross-sensory dysfunction, but rather is accounted for by sensorimotor integration deficits for vocal production and motor control due to cortico-basal ganglia pathology. This argument is supported by data from a recent study [19] that showed that the auditory event-related potentials (ERPs) were not different in PD vs. control subjects during listening to the playback of pitch shifted vocalization, supporting the notion that neural processing of auditory feedback error is not impaired in PD. However, when pitch shifts were delivered during vocalization, PD subjects 
exhibited a significant increase in the amplitude of auditory ERPs compared with controls [19]. This latter finding suggests that the pathologically increased gain of the state feedback controlling mechanisms is driven by top-down influence of the vocal motor system on auditory neural sensitivity that results in overshooting vocal compensation responses to feedback alterations in PD.

In this study, our data showed that STN-DBS improves vocal F0 motor control ability in subjects with PD, as reflected by the attenuation of compensation responses (i.e. dampening of 'overshooting' responses) to auditory feedback pitch-shift alterations during DBS ON vs. OFF. In addition, we found that STN-DBS was associated with reduced level of baseline (i.e. prestimulus) voice F0 perturbation as indexed by decreased jitter, and this effect was significantly correlated with DBS-induced attenuation of vocal compensation responses to auditory feedback pitch-shift stimuli. This observed correlational relationship was in line with data from previous studies [35-37] showing a direct correlation between the pathologically increased (i.e. overshooting) magnitude of vocal responses to auditory feedback alteration and higher voice F0 perturbation in subjects with PD. Based on findings of the present study, it can be suggested that STN-DBS improves vocal function by ameliorating motor symptoms related to lack of control over the laryngeal muscles for regulating voice F0. We suggest that DBS-induced improvement of voice F0 control results from the increased inhibitory effects of the basal ganglia on the cortical neural mechanisms of vocal motor control through stimulation of the hyperdirect (frontosubthalamic) pathways. However, our data did not reveal any significant effects of STN-DBS on other voice features such as HNR, intensity, or shimmer, suggesting that stimulation of the fronto-subthalamic pathways predominantly affect voice F0 motor control. Moreover, the absence of a significant correlation between the measures of vocal compensation and speech 
intelligibility scores indicates that DSB-induced improvement in some vocal motor control features (e.g., F0) may not necessarily translate into improvements in overall speech intelligibility. As mentioned earlier, it is reasonable to propose that such disconnected influences are accounted for by multiple factors including the inherent differences between the underlying mechanisms of voice vs. speech and the differential effects of STN-DBS on the mechanisms of vocal (i.e. phonatory) vs. oral (articulatory) motor control.

We observed that the effect of STN-DBS on attenuating vocal compensation responses to auditory feedback alteration was direction-specific and was only present when subjects increased their voice F0 in response to downward pitch-shift stimuli. However, no such modulatory effect of STN-DBS was observed when subjects decreased their voice F0 in response to upward pitch shifts in the auditory feedback. Data from previous studies have shown that vocal pitch motor control is mediated by complex patterns of laryngeal muscles contraction/relaxation that control the length, tension, and stiffness of vocal folds. In one study [38], it has been shown that increasing voice pitch in response to downward pitch-shift stimuli is facilitated by contraction of the cricothyroid (CT) and thyroarytenoid (TA) muscles, whereas decreasing voice pitch in response to upward stimuli is facilitated by relaxation of these muscles. However, as suggested by another study [39], contraction of the CT muscles did not always lead to raising voice pitch, but could also lower the pitch of the voice at low activation levels of the TA, lateral cricoarytenoid (LCA), and intra-arytenoid (IA) muscles. In addition, this latter study also showed that increasing TA activation was first accompanied by increased, and then decreased vocal pitch output at all activation levels of the CT, LCA, and IA muscles. Although the complex underlying mechanisms of vocal pitch motor control are not well-understood, data from the present study provide evidence for the differential effects of STN-DBS on the mechanisms of laryngeal muscle 
control for modulating voice F0 in response to auditory feedback pitch alterations. In the context of existing models of cortico-basal ganglia network [29-31], our data corroborate the notion that stimulation of the hyperdirect fronto-subthalamic pathway via STN-DBS inhibits cortical motor networks implicated in increasing voice F0, and subsequently, impedes PD subjects' abnormal (i.e. overshooting) vocal responses to downward auditory feedback pitch-shift stimuli. Another possible account of this observed effect is an overall DBS-induced increase in rigidity of the vocal fold muscles, which may subsequently impede the excessive increase in voice F0 and dampen the overshooting responses to downward pitch-shift stimuli in the auditory feedback. These findings indicate the positive impact of STN-DBS on specific aspects of voice motor control (i.e. F0); however, there is still a significant lack of knowledge about factors that contribute to improving the overall quality of speech in the context of a more general and complex sensorimotor system. In addition, understanding the effects of clinical and surgical factors (e.g., anatomical location of electrodes, stimulation amplitude, frequency, pulse width etc.) seem to be critical and warrants further investigations for predicting the effects of DBS on the outcome measures of voice and speech.

A potential limitation in the present study was the lack of matched control subjects for comparing their behavioral responses to PD subjects with bilateral STN-DBS implantation tested in this study. Although our study did not include a control group, comparing the data in our PD subjects with those tested using the same pitch shifting paradigm in previous studies [36,37] confirmed that the STN-DBS PD subjects in this study demonstrated vocal responses that were consistent with those in non-DBS PD subjects in previous studies. Namely, response magnitudes and latencies were consistent across DBS PD subjects in this study and non-DBS PD subjects in previous studies that used a similar AAF experimental paradigm. This also helped verify that 
relative to the matched control subjects in previous studies, the PD subjects with STN-DBS implantation in this study also generated abnormally excessive (i.e. overshooting) vocal responses to pitch shift stimuli, and our data provide the first evidence that DBS ON ameliorates this condition by attenuating vocal responses in a direction-specific manner only for downward pitch shifts in the auditory feedback. Furthermore, the absence of vocal response modulation for DBS ON vs. OFF for upward pitch-shift stimuli in this study served as a within-subject control factor, which further confirmed the effect of DBS on compensatory responses that raise voice F0 in response to downward pitch-shift alterations in the auditory feedback. A more comprehensive understanding about the effects of DBS warrants further investigations to provide more insights into the underlying neural mechanisms of voice and speech motor control.

\section{Authors' Roles}

R.B. and J.G. designed the research, recruited participants and collected data for the experiment. R.K., E.K., and N.N. assisted in data collection. Clinical assessment of PD and DBS setting management for all subjects were conducted by J.G. and N.N. (U. Iowa Department of Neurology and Neurosurgery). R.B. and K.J. analyzed the data and wrote the paper. All authors reviewed and approved the final draft.

\section{Financial Disclosures}

The authors declare no financial interest.

\section{References}

[1] Weaver FM, Stern M, Harris C, Jr WJM, Reda D, Moy CS, et al. Bilateral Deep Brain 
Disord 2007;13:S462-5. doi:10.1016/S1353-8020(08)70050-6.

[3] Skodda S, Grönheit W, Schlegel U, Südmeyer M, Schnitzler A, Wojtecki L. Effect of subthalamic stimulation on voice and speech in Parkinson's disease: For the better or worse? Front Neurol 2014;4 JAN:1-9. doi:10.3389/fneur.2013.00218.

[4] Skodda S. Effect of deep brain stimulation on speech performance in Parkinson's disease. Parkinsons Dis 2012;2012. doi:10.1155/2012/850596.

[5] Kleiner-Fisman G, Herzog J, Fisman DN, Tamma F, Lyons KE, Pahwa R, et al. Subthalamic nucleus deep brain stimulation: Summary and meta-analysis of outcomes. Mov Disord 2006;21:290-304. doi:10.1002/mds.20962.

[6] Piboolnurak P, Lang AE, Lozano AM, Miyasaki JM, Saint-Cyr JA, Poon YYW, et al. Levodopa response in long-term bilateral subthalamic stimulation for Parkinson's disease. Mov Disord 2007;22:990-7. doi:10.1002/mds.21482.

[7] Obeso JA, Olanow CW. Deep-Brain Stimulation of the Subthalamic Nucleus or the Pars. N Engl J Med 2001;345:956-63. doi:10.1056/NEJMoa000827.

[8] Dromey C, Kumar R, Lang AE, Lozano AM. An investigation of the effects of subthalamic nucleus stimulation on acoustic measures of voice. Mov Disord 2000;15:1132-8. doi:10.1002/1531-8257(200011)15:6<1132::AID-MDS1011>3.0.CO;2O.

[9] Gentil M, Pinto S, Pollak P, Benabid AL. Effect of bilateral stimulation of the subthalamic nucleus on parkinsonian dysarthria. Brain Lang 2003;85:190-6. doi:10.1016/S0093934X(02)00590-4. 
[10] Klostermann F, Ehlen F, Vesper J, Nubel K, Gross M, Marzinzik F, et al. Effects of subthalamic deep brain stimulation on dysarthrophonia in Parkinson's disease. J Neurol Neurosurg Psychiatry 2008;79:522-9. doi:10.1136/jnnp.2007.123323.

[11] Sidtis D, Sidtis JJ. Subcortical Effects on Voice and Fluency in Dysarthria: Observations from Subthalamic Nucleus Stimulation. J Alzheimer's Dis Park 2017;07. doi:10.4172/2161-0460.1000392.

[12] E. T, L. Z, I. M-T, E. F, S. P, T. F, et al. Effects of subthalamic stimulation on speech of consecutive patients with Parkinson disease. Neurology 2011;76:80-6. doi:10.1212/WNL.0b013e318203e7d0.

[13] Sidtis JJ, Alken AG, Tagliati M, Alterman R. Subthalamic Stimulation Reduces Vowel Space at the Initiation of Sustained Production : Implications for Articulatory Motor Control in Parkinson 's Disease 2016;6:361-70. doi:10.3233/JPD-150739.

[14] P. K, A. B, N. VB, S. C, V. F, C. A, et al. Five-Year Follow-up of Bilateral Stimulation of the Subthalamic Nucleus in Advanced Parkinson's Disease. N Engl J Med 2003;349:1925-34. doi:10.1056/NEJMoa035275.

[15] Fenoy AJ, McHenry MA, Schiess MC. Speech changes induced by deep brain stimulation of the subthalamic nucleus in Parkinson disease: involvement of the dentatorubrothalamic tract. J Neurosurg 2017;126:2017-27. doi:10.3171/2016.5.JNS16243.

[16] Tripoliti E, Limousin P, Foltynie T, Candelario J, Aviles-Olmos I, Hariz MI, et al. Predictive factors of speech intelligibility following subthalamic nucleus stimulation in consecutive patients with Parkinson's disease. Mov Disord 2014;29:532-8. doi:10.1002/mds.25816.

[17] Mollaei F, Shiller DM, Gracco VL. Sensorimotor adaptation of speech in Parkinson's 
disease. Mov Disord 2013;28:1668-74. doi:10.1002/mds.25588.

[18] Mollaei F, Shiller DM, Baum SR, Gracco VL. Sensorimotor control of vocal pitch and formant frequencies in Parkinson's disease. Brain Res 2016;1646:269-77. doi:10.1016/j.brainres.2016.06.013.

[19] Huang X, Chen X, Yan N, Jones JA, Wang EQ, Chen L, et al. The Impact of Parkinson 's Disease on the Cortical Mechanisms That Support Auditory - Motor Integration for Voice Control. Hum Brain Mapp 2016;4261:4248-61. doi:10.1002/hbm.23306.

[20] Baken, R.J., Orlikoff RF. Clinical measurement of speech and voice. 2nd Editio. San Diego: Singular Publishing Group, Inc.; 2000.

[21] Larson CR. Cross-modality influences in speech motor control: the use of pitch shifting for the study of F0 control. J Commun Disord 1998;31:489-502; quiz 502-3; 553.

[22] Benjamini Y, Hochberg Y. Controlling the false discovery rate a practical and powerful approach to multiple testing 1995:289-300.

[23] Ho AK, Bradshaw JL, Iansek R. For better or worse: The effect of Levodopa on speech in Parkinson's disease. Mov Disord 2008;23:574-80. doi:10.1002/mds.21899.

[24] Guenther FH, Ghosh SS, Tourville J a. Neural modeling and imaging of the cortical interactions underlying syllable production. Brain Lang 2006;96:280-301. doi:10.1016/j.bandl.2005.06.001.

[25] Houde JF, Nagarajan SS. Speech production as state feedback control. Front Hum Neurosci 2011;5:82. doi:10.3389/fnhum.2011.00082.

[26] Hickok G, Houde J, Rong F. Sensorimotor integration in speech processing: computational basis and neural organization. Neuron 2011;69:407-22. doi:10.1016/j.neuron.2011.01.019. 
[27] Hammer MJ, Barlow SM. Laryngeal somatosensory deficits in Parkinson's disease: Implications for speech respiratory and phonatory control. Exp Brain Res 2010;201:4019. doi:10.1007/s00221-009-2048-2.

[28] Larson CR, Altman KW, Liu H, Hain TC. Interactions between auditory and somatosensory feedback for voice F0control. Exp Brain Res 2008;187:613-21. doi:10.1007/s00221-008-1330-z.

[29] Aron AR, Durston S, Eagle DM, Logan GD, Stinear CM, Stuphorn V. Converging Evidence for a Fronto-Basal-Ganglia Network for Inhibitory Control of Action and Cognition. J Neurosci 2007;27:11860-4. doi:10.1523/JNEUROSCI.3644-07.2007.

[30] Jahfari S, Waldorp L, van den Wildenberg WPM, Scholte HS, Ridderinkhof KR, Forstmann BU. Effective Connectivity Reveals Important Roles for Both the Hyperdirect (Fronto-Subthalamic) and the Indirect (Fronto-Striatal-Pallidal) Fronto-Basal Ganglia Pathways during Response Inhibition. J Neurosci 2011;31:6891-9. doi:10.1523/JNEUROSCI.5253-10.2011.

[31] Aron AR. Cortical and Subcortical Contributions to Stop Signal Response Inhibition: Role of the Subthalamic Nucleus. J Neurosci 2006;26:2424-33. doi:10.1523/JNEUROSCI.4682-05.2006.

[32] Chang EF, Niziolek C a, Knight RT, Nagarajan SS, Houde JF. Human cortical sensorimotor network underlying feedback control of vocal pitch. Proc Natl Acad Sci U S A 2013;110:2653-8. doi:10.1073/pnas.1216827110.

[33] Behroozmand R, Oya XH, Nourski K V, Kawasaki H, Larson CR, Brugge JF, et al. Neural Correlates of Vocal Production and Motor Control in Human Heschl 's Gyrus. J Neurosci 2016;36:2302-15. doi:10.1523/JNEUROSCI.3305-14.2016. 
[34] Houde JF, Chang EF. The cortical computations underlying feedback control in vocal production. Curr Opin Neurobiol 2015;33:174-81. doi:10.1016/j.conb.2015.04.006.

[35] Chen X, Zhu X, Wang EQ, Chen L, Li W, Chen Z, et al. Sensorimotor control of vocal pitch production in Parkinson's disease. Brain Res 2013;1527:99-107.

doi:10.1016/j.brainres.2013.06.030.

[36] Liu H, Wang EQ, Metman LV, Larson CR. Vocal responses to perturbations in voice auditory feedback in individuals with parkinson's disease. PLoS One 2012;7. doi:10.1371/journal.pone.0033629.

[37] Huang X, Chen X, Yan N, Jones JA, Wang EQ, Chen L, et al. The Impact of Parkinson 's Disease on the Cortical Mechanisms That Support Auditory - Motor Integration for Voice Control. Hum Brain Mapp 2016. doi:10.1002/hbm.23306.

[38] Liu H, Behroozmand R, Bove M, Larson CR. Laryngeal electromyographic responses to perturbations in voice pitch auditory feedback. J Acoust Soc Am 2011;129:3946-54. doi:10.1121/1.3575593.

[39] Chhetri DK, Neubauer J, Sofer E, Berry DA. Influence and interactions of laryngeal adductors and cricothyroid muscles on fundamental frequency and glottal posture control. J Acoust Soc Am 2014;135:2052-64. doi:10.1121/1.4865918. 


\section{Figure captions}

Figure 1. Panels A-C: the mean of vocalization F0, intensity, and HNR across all trials overlaid for DBS ON vs. DBS OFF in one representative subject (shaded areas show the standard deviation; time 0 denotes onset of vocalization). Panels D-H: bar plot representations of the mean and SEM of the grand-average ( $n=10$ subjects) measures of vocalization F0, intensity, HNR, jitter, and shimmer for DBS ON vs. DBS OFF.

Figure 2. Panels A-C: Vocal compensation responses to upward pitch-shift stimuli (+100 cents). Panel A: profiles of grand-average mean voice F0 responses overlaid for DBS ON vs. OFF (time 0 notes onset of pitch-shift stimuli). Panel B: bar plot representation of the grand-average response means within a $200 \mathrm{~ms}$ window centered on the peak. Panel C: correlation plots of vocal compensation vs. jitter modulation during DBS ON vs. DBS OFF. Panels D-F Results for vocal compensations to downward pitch-shift stimuli (-100 cents). All error bars represent the standard error of the mean $\left(\mathrm{SE}_{\mathrm{M}}\right)$. 
Figure 3. The sensorimotor integration model of vocal control. In this model, the auditory-motor interface transforms efference copies of motor plans into forward predictions and compares them with auditory feedback to detect and correct for errors through generating compensatory vocal motor responses. In Parkinson's disease, dysfunctions in cortico-basal ganglia network results in reduced inhibitory input to cortical motor areas. This reduced inhibition contributes to increased corrective efforts in the feedforward motor system leading to abnormal (overshooting) vocal compensation responses to alterations in the auditory feedback (AAF). In addition, reduced inhibition of the vocal motor cortex increases its top-down effect on enhancing auditory neural sensitivity to feedback alterations. This increased neural sensitivity results in elevated sensory gain for generating larger error signals, and subsequently, larger compensatory vocal responses to alterations in the auditory feedback.

vPMC: ventral pre-motor cortex; IFG: inferior frontal gyrus; M1: primary motor cortex; HG: Heschl's gyrus; STG: superior temporal gyrus; STS: superior temporal sulcus; Spt: Sylvian parietal temporal; PT; planum temporale 
Table 1. Subjects' demographic and clinical assessment data

\begin{tabular}{|c|c|c|c|c|c|c|c|c|c|}
\hline $\begin{array}{l}\text { Subj. } \\
\text { ID }\end{array}$ & $\begin{array}{c}\text { Age/ } \\
\text { Sex }\end{array}$ & $\begin{array}{l}\text { PD } \\
\text { Duration } \\
\text { (yrs) }\end{array}$ & $\begin{array}{l}\text { Time } \\
\text { from DBS } \\
\text { Surgery to } \\
\text { Testing } \\
\text { (yrs) }\end{array}$ & Medications & LED & $\begin{array}{c}\text { Left } \\
\text { stimulation } \\
\text { setting } \\
(\text { contact } \#, \\
\text { voltage }(\mathrm{V}), \\
\text { pulse width } \\
(\mu \mathrm{sec}) / \\
\text { frequency } \\
(\mathrm{Hz})) \\
\end{array}$ & $\begin{array}{c}\text { Right } \\
\text { stimulation } \\
\text { setting } \\
\text { (contact \#, } \\
\text { voltage }(\mathrm{V}), \\
\text { pulse width } \\
(\mu \mathrm{sec}) / \\
\text { frequency } \\
(\mathrm{Hz})) \\
\end{array}$ & $\begin{array}{l}\text { UPDRS } \\
\text { Part III } \\
\text { (DBS } \\
\text { OFF/ } \\
\text { ON) }\end{array}$ & VHI \\
\hline 1 & $59 / \mathrm{F}$ & 6 & 1.1 & $\begin{array}{l}\text { (1) Amantadine } 100 \mathrm{mg} \text { bid } \\
\text { (2) Comtan } 200 \mathrm{mg} \text { qid } \\
\text { (3) Sinemet } 50-200 \mathrm{mg} \times 11 / \mathrm{d}\end{array}$ & 1650 & $\begin{array}{l}\text { Case }+2-, 1 \\
\quad 60 / 130\end{array}$ & $\begin{array}{c}\text { Case + 10-, } \\
1,60 / 130\end{array}$ & $43 / 26$ & 60 \\
\hline 2 & $62 / \mathrm{M}$ & 12 & 3.1 & (1) Xanax $1 \mathrm{mg}$ prn & 0 & $\begin{array}{l}\text { Case }+(1,2)- \\
, 3.5,60 / 150\end{array}$ & $\begin{array}{c}\text { Case + } \\
(9,10)-, 3.8 \\
70 / 150\end{array}$ & $31 / 19$ & 83 \\
\hline 3 & $66 / M$ & 16 & 0.6 & $\begin{array}{l}\text { (1) Amantadine } 100 \mathrm{mg} \text { tid } \\
\text { (2) Klonopin } 0.5 \mathrm{mg} \text { qhs } \\
\text { (3) Requip } 2 \mathrm{mg} \text { tid } \\
\text { (4) Requip } 5 \mathrm{mg} \text { qid } \\
\text { (5) Rytary } 48.75-195 \mathrm{mg} \times 13 / \mathrm{d} \\
\text { (6) Xanax } 0.25 \mathrm{mg} \text { tid }\end{array}$ & 2341 & $\begin{array}{l}\text { Case }+8- \\
1.6,60 / 135\end{array}$ & $\begin{array}{l}\text { Case + 9- } \\
1.5,60 / 135\end{array}$ & $23 / 7$ & 52 \\
\hline 4 & $76 / \mathrm{M}$ & 8 & 1.6 & $\begin{array}{l}\text { (1) Namenda } 28 \mathrm{mg} \text { qd } \\
\text { (2) Sinemet } 25-100 \mathrm{mg} \text { half qid }\end{array}$ & 200 & $\begin{array}{l}\text { Case }+2- \\
1.1,60 / 120\end{array}$ & $\begin{array}{l}\text { Case }+10- \\
1.1,60 / 135\end{array}$ & $27 / 24$ & 68 \\
\hline 5 & $61 / \mathrm{F}$ & 11 & 2.6 & $\begin{array}{l}\text { (1) Amantadine } 100 \mathrm{mg} \text { bid } \\
\text { (2) Azilect } 1 \mathrm{mg} \mathrm{qd} \\
\text { (3) Requip } 8 \mathrm{mg} \mathrm{qd} \\
\text { (4) Sinemet } 50-200 \mathrm{mg} \text { tid }\end{array}$ & 1260 & $\begin{array}{l}\text { Case }+1- \\
1.7,60 / 135\end{array}$ & $\begin{array}{l}(8,10)+9- \\
2.2,60 / 135\end{array}$ & $9 / 3$ & 15 \\
\hline 6 & $51 / \mathrm{F}$ & 12 & 1.7 & $\begin{array}{l}\text { (1) Klonopin } 1 \mathrm{mg} \text { bid } \\
\text { (2) Sinemet } 25-100 \mathrm{mg} \mathrm{x} 9 / \mathrm{d}\end{array}$ & 900 & $\begin{array}{c}\text { Case }+2- \\
2.2,120 / 130\end{array}$ & $\begin{array}{l}\text { Case + 10-, } \\
2.7,140 / 130\end{array}$ & $31 / 11$ & 32 \\
\hline 7 & $68 / \mathrm{M}$ & 14 & 1.1 & $\begin{array}{l}\text { (1) Parcopa } 25-100 \mathrm{mg} \text { prn } \\
\text { (2) Rytary } 23.75-95 \mathrm{mg} \mathrm{x} 8 / \mathrm{d}\end{array}$ & 656 & $\begin{array}{c}3+2-, 3.6 \\
140 / 130\end{array}$ & $\begin{array}{c}3+2-, 3.5 \\
100 / 130\end{array}$ & $8 / 2$ & 30 \\
\hline 8 & $64 / \mathrm{M}$ & 9 & 2.5 & $\begin{array}{l}\text { (1) Amantadine } 100 \mathrm{mg} \text { qd } \\
\text { (2) Klonopin } 0.5 \mathrm{mg} \text { half tid } \\
\text { (3) Stalevo } 18.75-75-200 \mathrm{mg} \\
\text { x } 5 / \mathrm{d}\end{array}$ & 665 & $\begin{array}{l}\text { Case }+8- \\
3.7,120 / 130\end{array}$ & $\begin{array}{l}\text { Case }+0- \\
3.6,120 / 130\end{array}$ & $34 / 24$ & 68 \\
\hline 9 & $72 / \mathrm{M}$ & 10 & 1.2 & $\begin{array}{l}\text { (1) Amantadine } 100 \mathrm{mg} \text { bid } \\
\text { (2) Sinemet } 25-100 \mathrm{mg} \mathrm{x6} / \mathrm{d}\end{array}$ & 700 & $\begin{array}{l}\text { Case }+3- \\
2.7,60 / 130\end{array}$ & $\begin{array}{l}\text { Case }+8- \\
3.5,90 / 130\end{array}$ & $21 / 8$ & 41 \\
\hline 10 & $69 / F$ & 13 & 3.4 & $\begin{array}{l}\text { (1) Sinemet } 25-100 \text { bid } \\
\text { (2) Xanax } 0.5 \mathrm{mg} \text { prn }\end{array}$ & 200 & $\begin{array}{l}\text { Case }+0- \\
2.5,60 / 130\end{array}$ & $\begin{array}{l}\text { Case + 10- } \\
2.5,60 / 130\end{array}$ & $21 / 16$ & 25 \\
\hline
\end{tabular}

LED: Levodopa Equivalent Dose. UPDRS: Unified Parkinson's Disease Rating Scale. VHI: Voice Handicap Index (higher numbers indicate greater subjective impairment). 

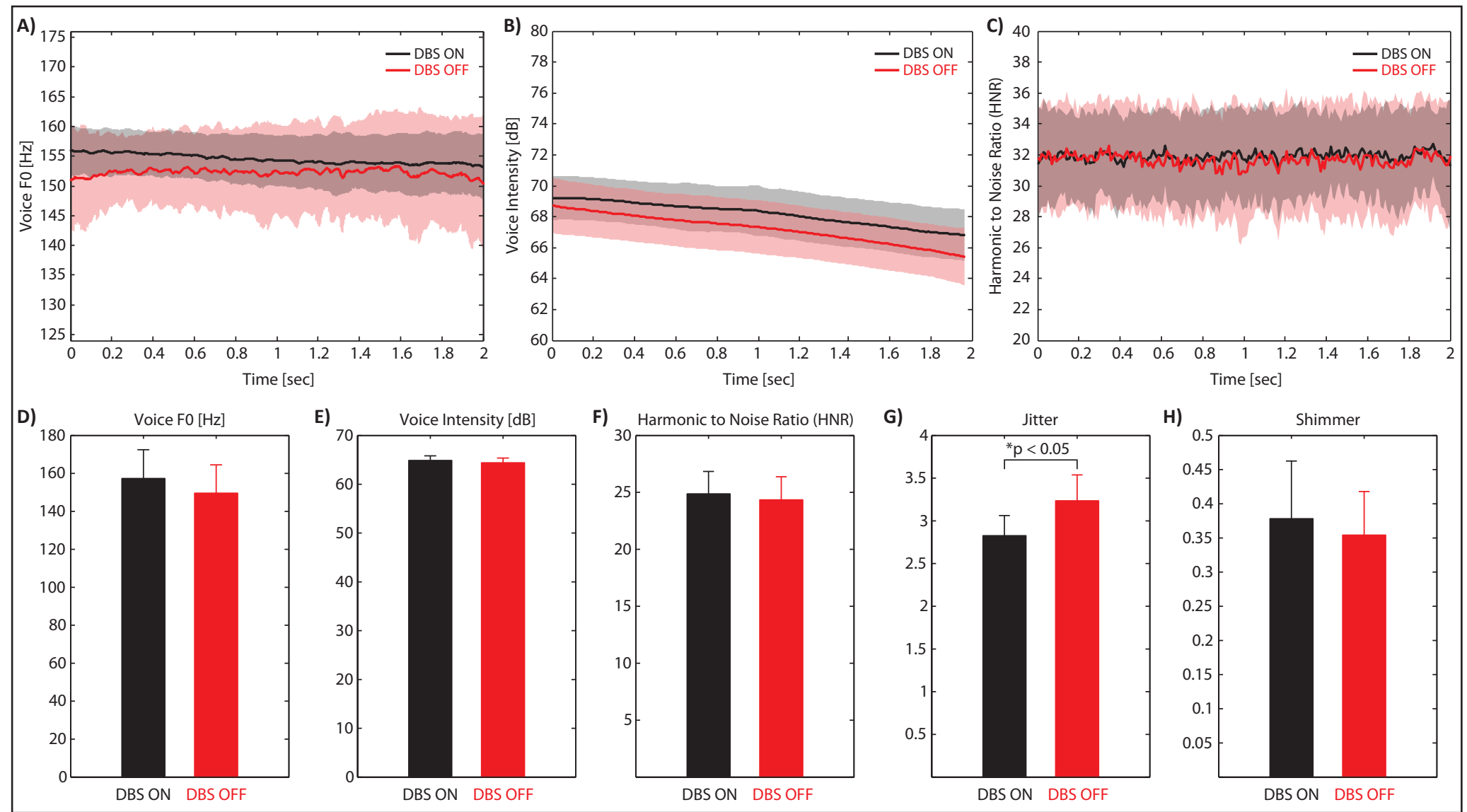

DBS ON DBS OFF

DBS ON DBS OFF 
Figure 2
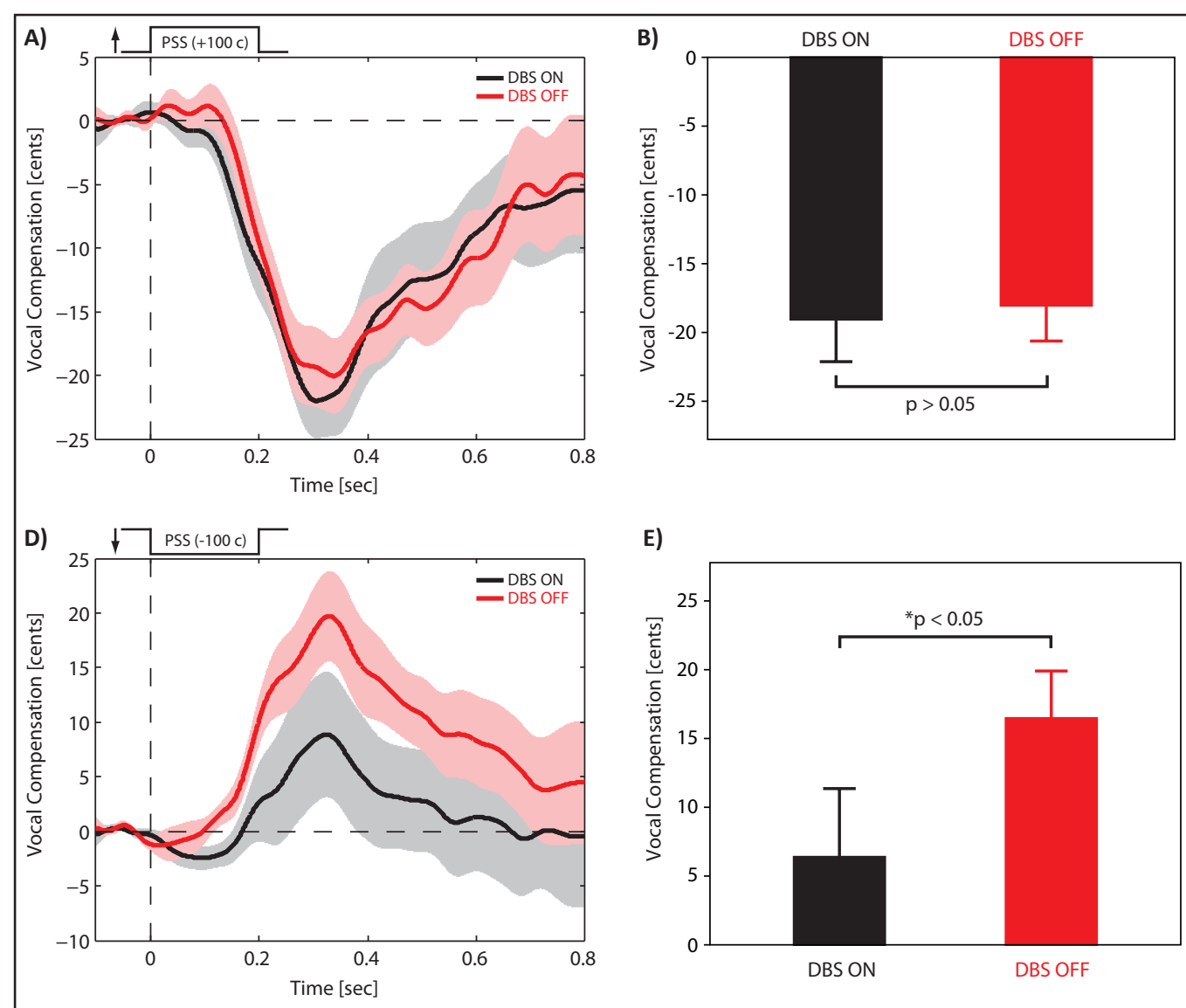

E)

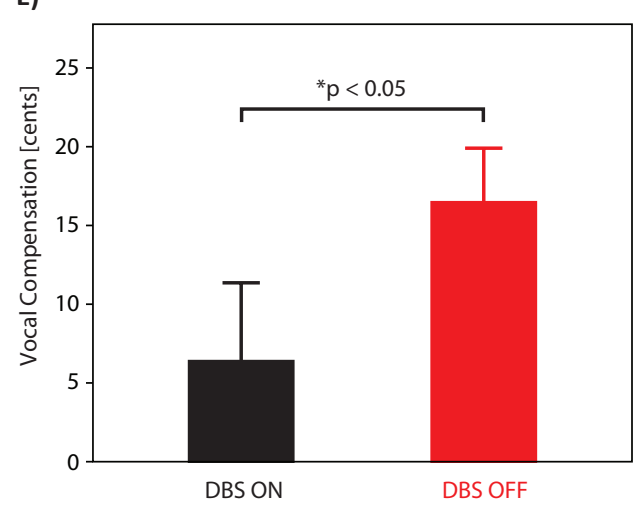

C) 0.4 DBS Modulation (DBS ON - DBS OFF)

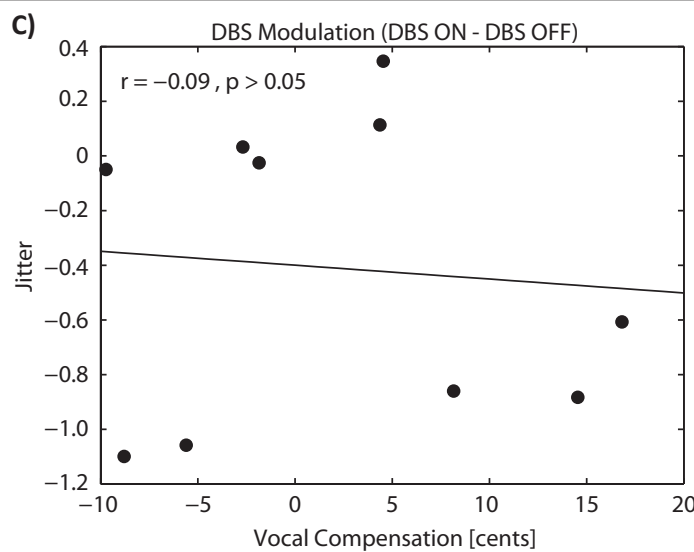

F)

F) DBS Modulation (DBS ON - DBS OFF)

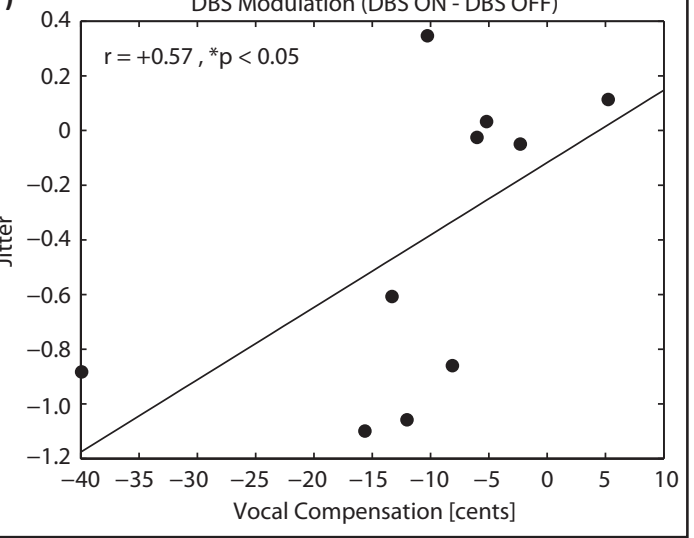




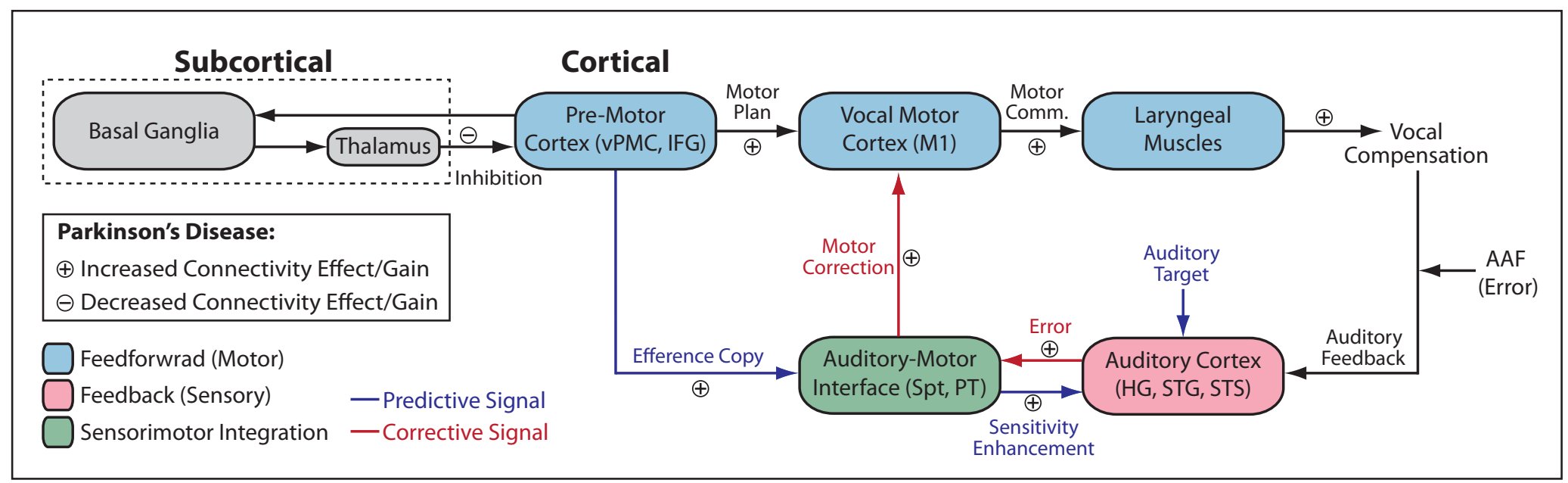

